\title{
FACTORIZATION OF PROBABILITY MEASURES AND ABSOLUTELY MEASURABLE SETS
}

\author{
DAVID BLACKWELL AND ASHOK MAITRA
}

\begin{abstract}
We find necessary and sufficient conditions for a separable metric space $Y$ to possess the property that for any measurable space $(X, A)$ and probability measure $P$ on $X \times Y, P$ can be factored.
\end{abstract}

1. Introduction. We characterize separable metric spaces $Y$ which have the following property: for any measurable space $(X, \mathcal{A})$ and any probability measure $P$ on the product space $(X \times Y, A \times B(Y))$, where $B(Z)$ will denote the Borel $\sigma$-field of a metric space $Z, P$ can be factored: $P=\mathcal{Q} \times T$, where $\mathcal{Q}$ is a probability measure on $(X, A)$ and $T: X \times B(Y) \rightarrow[0,1]$ is an $A$-measurable transition function such that

$$
P(A \times B)=\int_{A} T(B \mid x) d Q(x)
$$

for every $A \in A$ and $B \in B(Y)$.

The factorability of $P$ is of obvious interest to the Bayesian statistician. For, imagine that $(Y, B(Y))$ is the parameter space and that $(X, A)$ is the sample space. A prior on $(Y, B(Y))$ together with a model (that is, a $B(Y)$-measurable transition function $T: Y \times A \rightarrow[0,1])$ determine a probability measure $P$ on $(X \times Y, A \times B(Y))$. The Bayesian bases his inference on the posterior distribution of the parameter given the sample, so he needs to factor $P$ as above.

The main result of the paper is as follows.

THEOREM. Let $Y$ be a separable metric space. Then the following conditions on $Y$ are equivalent.

(a) $Y$ is absolutely measurable, i.e., if $\tilde{Y}$ is a metric completion of $Y$ and $\lambda$ is a probability measure on the Borel $\sigma$-field of $\tilde{Y}$, then $Y$ is $\lambda$-measurable.

(b) For any measurable space $(X, A)$ and any probability measure $P$ on $(X \times$ $Y, A \times B(Y)), P$ can be factored.

(c) For any Polish space $X$ and any probability measure $P$ on $(X \times Y, B(X) \times$ $B(Y)), P$ can be factored.

The above result gives a new characterization of absolutely measurable sets. Using the theory of compact measures developed by Marczewski [3], one can deduce the implication $(a) \rightarrow(b)$ from a result of Jirina [1] and the implication (b) $\rightarrow(a)$ from a result of Pachl [4]. The implication (c) $\rightarrow(\mathrm{a})$ is new. Our proofs, however, are direct and elementary in nature and do not use the somewhat elaborate machinery of compact measures.

The proofs will be given in $\S 3$. $§ 2$ explains the notation to be used.

Received by the editors July 5, 1983 and, in revised form, October 24, 1983.

1980 Mathematics Subject Classification. Primary 28A05, 60A10, 60A05; Secondary 28A50.

Key words and phrases. Probability measure, product space, transition function, factorization, absolutely measurable set, prior distribution, posterior distribution. 
2. Notation. If $(X, \mathcal{A})$ is a measurable space, $M(X, \mathcal{A})$ will denote the set of probability measures on $(X, A)$. If $(Z, C)$ is another measurable space, a function $T: X \times C \rightarrow[0,1]$ is said to be a $(X, A)-(Z, C)$ transition function if, for each $x \in X, T(\cdot \mid x)$ is a probability measure on $(Z, C)$, and for each $C \in C, T(C \mid \cdot)$ is an $A$ measurable function on $X$. The set of $(X, A)-(Z, C)$ transition functions is denoted by $M(Z, C \mid X, A)$. If $X, Z$ are metric spaces, we write $M(X)$ for $M(X, B(X))$ and $M(Z \mid X)$ for $M(Z, B(Z) \mid X, B(X))$.

We denote by $2^{N}$ the set of infinite sequences of 0 's and 1's and by $S$ the set of finite sequences of 0 's and 1's of positive length. For each $\mathfrak{s} \in S, L(\mathfrak{s})$ will denote the set of elements of $2^{N}$ that begin with $\mathfrak{s}$. The sets $L(\mathfrak{s}), \mathfrak{s} \in S$, form a base for a topology on $2^{N}$. Endowed with this topology, $2^{N}$ is a homeomorph of the Cantor ternary set.

If $Z$ is a metric space and $\lambda \in M(Z), \lambda^{*}$ and $\lambda_{*}$ denote, respectively, the outer measure and inner measure induced by $\lambda$.

3. Proofs. We begin with two lemmas, both of which are well known, and only the second will be proved here.

LEMMA 1. Suppose $f: S \rightarrow[0,1]$ satisfies (i) $f(0)+f(1)=1$, and (ii) $f(\mathfrak{s 0})+$ $f(\mathfrak{s} 1)=f(\mathfrak{s})$ for each $\mathfrak{s} \in S$. Then there is a unique $\mu \in M\left(2^{N}\right)$ such that $\mu(L(\mathfrak{s}))=f(\mathfrak{s})$ for each $\mathfrak{s} \in S$.

LEMMA 2. Suppose $X$ is a Polish space and let $V_{\mathfrak{n}}, \mathfrak{n} \geq 1$, be a base for the topology of $X$. Let $\varphi: X \rightarrow 2^{N}$ be defined by: $\varphi(x)=\left(I_{V_{1}}(x), I_{V_{2}}(x), \ldots\right)$, where $I_{V_{i}}$ denotes the indicator function of the set $V_{i}$. Then $\varphi$ is a Borel isomorphism of $X$ and $\varphi(X)$, and $\varphi(X)$ is Borel in $2^{N}$ (indeed, $a G_{\delta}$ in $2^{N}$ ).

PROOF. Since the sets $V_{\mathfrak{n}}$ separate points of $X$, the function $\varphi$ is one-one. It is easy to see that the inverse image of an open set under $\varphi$ is a $F_{\sigma}$ set in $X$ and the forward image of an open set under $\varphi$ is open in $\varphi(X)$. In other words, $\varphi^{-1}$ is continuous. So by a well-known result [2, Corollary 3 , p. 436$]$, there is a $G_{\delta}$ subset $G$ of $2^{N}$ and a continuous function $\mathfrak{g}: G \rightarrow X$ such that $\varphi(X) \subseteq G$ and $\mathfrak{g}=\varphi^{-1}$ on $\varphi(X)$. Consequently, $\varphi(X)=\{\mathfrak{z} \in G: \varphi(\mathfrak{g}(\mathfrak{z}))=\mathfrak{z}\}$. Hence, if $W_{\mathfrak{n}}, \mathfrak{n} \geq 1$, is a base for the topology of $G$, then

$$
G \backslash \varphi(X)=\bigcup_{\mathfrak{n} \geq 1}\left[\mathfrak{g}^{-1}\left(\varphi^{-1}\left(W_{\mathfrak{n}}\right)\right) \cap\left(G \backslash W_{\mathfrak{n}}\right)\right],
$$

so that $G \backslash \varphi(X)$ is a $F_{\sigma}$ set in $G$. It follows that $\varphi(X)$ is a $G_{\delta}$ in $G$, so a $G_{\delta}$ in $2^{N}$. The second sentence of the proof already establishes that $\varphi$ is a Borel isomorphism of $X$ and $\varphi(X)$.

We now turn to the proof of the Theorem. The implication $(a) \rightarrow(b)$ is proved first for $Y$ a Borel subset of $2^{N}$, then for $Y$ a Borel subset of a Polish space, and finally for $Y$ an absolutely measurable set.

Let, then, $Y$ be a Borel subset of $2^{N}$. Suppose $P$ is a probability measure on $(X \times Y, A \times B(Y))$. We define $P^{\prime}$ on $\left(X \times 2^{N}, A \times B\left(2^{N}\right)\right)$ by setting $P^{\prime}(E)=$ $P(E \cap(X \times Y))$ for $E \in A \times B\left(2^{N}\right)$, so $P^{\prime}$ is a probability measure. For each $\mathfrak{s} \in S$, fix a version $P^{\prime}\left(L(\mathfrak{s}) \mid A^{*}\right)$ of the conditional probability under $P^{\prime}$ of the set $X \times L(\mathfrak{s})$ given $A^{*}$, where $A^{*}$ is the $\sigma$-field on $X \times 2^{N}$ of events of the form $A \times 2^{N}$ with $A \in A$. For each $x \in \mathcal{X}$ and $\mathfrak{s} \in S$ set

$$
\mathfrak{h}(\mathfrak{s}, x)=P^{\prime}\left(L(\mathfrak{s}) \mid \mathcal{A}^{*}\right)(x) .
$$


It is easy to verify using the properties of conditional probabilities that there is a set $H \in A$ with $P^{\prime}\left(H \times 2^{N}\right)=0$ such that whenever $x \notin H, \mathfrak{h}(0, x)+\mathfrak{h}(1, x)=1$ and $\mathfrak{h}(\mathfrak{s} 0, x)+\mathfrak{h}(\mathfrak{s} 1, x)=\mathfrak{h}(\mathfrak{s}, x)$ for $\mathfrak{s} \in S$. Redefine $\mathfrak{h}(\mathfrak{s}, x)$ to be $P^{\prime}(X \times L(\mathfrak{s}))$ for $x \in H$ and $\mathfrak{s} \in S$. Then, for each $x \in X$, the function $\mathfrak{h}(\cdot, x)$ satisfies the hypotheses of Lemma 1 , so that there is a unique probability measure $T^{\prime}(\cdot \mid x)$ on $B\left(2^{N}\right)$ such that $T^{\prime}(L(\mathfrak{s}) \mid x)=\mathfrak{h}(\mathfrak{s}, x)$ for all $\mathfrak{s} \in S$. Since $\mathfrak{h}(\mathfrak{s}, \cdot)$ is $A$-measurable on $X$, so is $T^{\prime}(L(\mathfrak{s}) \mid \cdot)$ for each $\mathfrak{s} \in S$, whence, by a routine measure-theoretic argument, $T^{\prime}(B \mid \cdot)$ is $A$-measurable for every $B \in B\left(2^{N}\right)$. Denoting by $\mathcal{Q}$ the marginal of $P^{\prime}$ on $X$, we have for each $\mathfrak{s} \in S$ and $A \in A$,

$$
\begin{aligned}
P^{\prime}(A \times L(\mathfrak{s})) & =\int_{A} P^{\prime}\left(L(\mathfrak{s}) \mid \mathcal{A}^{*}\right)(x) d \mathcal{Q}(x) \\
& =\int_{A} \mathfrak{h}(\mathfrak{s}, x) d \mathcal{Q}(x) \quad\left(\text { as } \mathfrak{h}(\mathfrak{s}, \cdot)=P^{\prime}\left(L(\mathfrak{s}) \mid \mathcal{A}^{*}\right)(\cdot) \text { a.s. }(\mathcal{Q})\right) \\
& =\int_{A} T^{\prime}(L(\mathfrak{s}) \mid x) d \mathcal{Q}(x),
\end{aligned}
$$

so again by a routine measure-theoretic argument

$$
P^{\prime}(A \times B)=\int_{A} T^{\prime}(B \mid x) d \mathcal{Q}(x)
$$

for each $B \in B\left(2^{N}\right)$. Since $P^{\prime}(X \times Y)=1$, it is easily seen that there is set $H^{\prime} \in A$ such that $\mathcal{Q}\left(H^{\prime}\right)=0$ and $T^{\prime}(Y \mid x)=1$ for all $x \in X \backslash H^{\prime}$. Finally, define for any $B \in B(Y)$,

$$
T(B \mid x)= \begin{cases}T^{\prime}(B \mid x) & \text { if } x \in X \backslash H^{\prime}, \\ P(X \times B) & \text { if } x \in H^{\prime}\end{cases}
$$

Then $T \in M(Y, B(Y) \mid X, \mathcal{A})$ and $P=\underline{Q} \times T$.

Consider next the case where $Y$ is a Borel subset of a Polish space $Z$. If $V_{\mathfrak{n}}, \mathfrak{n} \geq 1$, is a base for the toplogy of $Z$, we define $\varphi: Z \rightarrow 2^{N}$ by $\varphi(z)=\left(I_{V_{1}}(z), I_{V_{2}}(z), \ldots\right)$. By Lemma 2, $\varphi$ is a Borel isomorphism of $Z$ and $\varphi(Z)$, and $\varphi(Z)$ is Borel in $2^{N}$. If we now restrict $\varphi$ to $Y$, then (i) $\varphi(Y)$ is a Borel subset of $2^{N}$, and (ii) $\varphi$ sets up a Borel isomorphism of $Y$ and $\varphi(Y)$. The probability measure $P \in M(X \times Y, A \times$ $B(Y))$ can now be taken over to $(X \times \varphi(Y), A \times B(\varphi(Y)))$, factored by virtue of the previous argument, and the factorization brought back to $(X \times Y, A \times B(Y))$, again using the function $\varphi$.

Lastly, let $Y$ be absolutely measurable and let $P \in M(X \times Y, A \times B(Y))$. Denote by $\mu$ the marginal of $P$ on $Y$. As $Y$ is absolutely measurable, there is $Y^{*} \subseteq Y$ such that $Y^{*}$ is a Borel subset of a Polish space and $\mu\left(Y^{*}\right)=1$. It follows that $P\left(X \times Y^{*}\right)=1$. Restrict $P$ to $\left(X \times Y^{*}, A \times B\left(Y^{*}\right)\right)$, call it $P^{\prime}$. By the previous paragraph, $P^{\prime}$ can be factored: $P^{\prime}=\mathcal{Q} \times T^{\prime}$, where $\mathcal{Q} \in M(X, \mathcal{A})$ and $T^{\prime} \in$ $M\left(Y^{*}, B\left(Y^{*}\right) \mid X, A\right)$. Set $T(B \mid x)=T^{\prime}\left(B \cap Y^{*} \mid x\right)$ for $B \in B(Y), x \in X$. Then $T \in M(Y, B(Y) \mid X, A)$ and $P=\mathcal{Q} \times T$, completing the proof of $(\mathrm{a}) \rightarrow(\mathrm{b})$. (b) $\rightarrow(\mathrm{c})$ is trivial.

To prove $(\mathrm{c}) \rightarrow(\mathrm{a})$, assume $Y$ is a separable metric space, and let $X$ be a metric completion of $Y$. Suppose $\lambda \in M(X)$. If $\lambda^{*}(Y)=0, Y$ is $\lambda$-measurable. So assume $\lambda^{*}(Y)>0$, and further, by normalizing $\lambda$ if necessary, assume without loss of generality that $\lambda^{*}(Y)=1$. Let $C$ be the smallest $\sigma$-field on $X$ containing 
$B(X) \cup\{Y\}$. Extend $\lambda$ to a measure $\mu$ on $C$ by setting

$$
\mu\left(\left(E_{1} \cap Y\right) \cup\left(E_{2} \cap(X \backslash Y)\right)\right)=\lambda\left(E_{1}\right), \quad E_{1}, E_{2} \in B(X) .
$$

We define a probability measure $P$ on $(X \times X, B(X) \times C)$ by

$$
P(A \times B)=\int_{B} I_{A}(\mathfrak{y}) d \mu(\mathfrak{y}), \quad A \in B(X), B \in \mathcal{C},
$$

so that $P(A \times B)=\mu(A \cap B)$.

Observe that the trace $C \cap Y$ of the $\sigma$-field $C$ on $Y$ is just $B(Y)$, so the trace of $B(X) \times C$ on $X \times Y$ is $B(X) \times B(Y)$. Furthermore, $\mu(Y)=1$ and hence $P(X \times Y)=1$. Let $P^{\prime}$ denote the restriction of $P$ to $(X \times Y, B(X) \times B(Y))$, so that $P^{\prime} \in M(X \times Y)$. By the hypothesis of (c), we can factor $P^{\prime}: P^{\prime}=\mathcal{Q} \times T^{\prime}$, where $\mathcal{Q} \in M(X)$ and $T^{\prime} \in M(Y \mid X)$. We define $T(C \mid x)=T^{\prime}(C \cap Y \mid x)$ for $C \in \mathcal{C}, x \in X$. It is now easy to verify that $T \in M(X, C \mid X, B(X))$ and that $P=\mathcal{Q} \times T$.

Next observe that for $A \in B(X), B \in C$,

$$
\mu(A \cap B)=P(A \times B)=\int_{A} T(B \mid x) d \mathcal{Q}(x) .
$$

Setting $B=X$ in $(*)$, we get $\mu(A)=\mathcal{Q}(A)$, so $\mathcal{Q}=\lambda$. Setting $A=B$ in $(*)$, we have

$$
\lambda(A)=\int_{A} T(A \mid x) d \lambda(x) .
$$

Since $0 \leq T(A \mid x) \leq 1$, this implies for each $A \in B(X)$ that $T(A \mid x)=1$ a.s. $(\lambda)$ on $A$. So, if $\left\{G_{\mathfrak{n}}, \mathfrak{n} \geq 1\right\}$ is a countable generating class for $B(X)$, then there is a set $K \in B(X)$ with $\lambda(K)=0$ such that whenever $x \in X \backslash K$

$$
T\left(G_{\mathfrak{n}} \mid x\right)=1 \quad \text { if } x \in G_{\mathfrak{n}} \quad \text { and } \quad T\left(X \backslash G_{\mathfrak{n}} \mid x\right)=1 \quad \text { if } x \notin G_{\mathfrak{n}} .
$$

It follows immediately that for $x \notin K, T(\{x\} \mid x)=1$. By construction of $T$, we have $T(Y \mid x)=1$ for all $x \in X$. It follows that $X \backslash K \subset Y$, so that $\lambda_{*}(Y)=1$, and hence $Y$ is $\lambda$-measurable. This completes the proof.

\section{REFERENCES}

1. M. Jirina, Conditional probabilities on $\sigma$-algebras with countable basis, Czechoslovak. Math. J. 4 (1954), 372-380; English transl., Selected Transl. Math. Statist. Prob., Vol. 2, Amer. Math. Soc., Providence, R.I., 1962, pp. 79-86.

2. K. Kuratowski, Topology, Vol. 1, 5th ed., PWN, Warsaw; Academic Press, New York; "Mir", Moscow, 1966.

3. E. Marczewski, On compact measures, Fund. Math. 40 (1953), 113-124.

4. Jan K. Pachl, Disintegration and compact measures, Math. Scand. 43 (1978), 157-168. 94720

Department of Statistics, University of CALifornia, Berkeley, California

Statistics-Mathematics Division, Indian Statistical Institute, Calcutta700035 , INDIA 